\title{
Estudios superiores y trabajo: implicancias de su simultaneidad
}

Study and work: implications of their simultaneity

\author{
Andrea Pacífico \\ Pablo Héctor Gandolfo \\ Facultad de Ciencias Económicas, \\ Universidad Nacional del Litoral. Argentina \\ E-mail: andpacifico@yahoo.com.ar \\ E-mail:pablog_008@hotmail.com
}

Fecha de recepción: 03/03/2016 Fecha de aceptación: 25/04/2016

Palabras clave - estudios superiores

- trabajo

- desempeño académico

- horas semanales de trabajo

- carreras de ciencias económicas

\section{Resumen}

El objetivo de este artículo es determinar cómo la simultaneidad entre estudios superiores y trabajo inciden en las trayectorias de los estudiantes avanzados de la Facultad de Ciencias Económicas de la Universidad Nacional del Litoral (FCE-UNL). Para cumplir con este objetivo, se utilizó información de una encuesta realizada en 2015 a los alumnos de la FCE-UNL, junto con datos oficiales de dicha institución. Este estudio muestra que los trabajos que los alumnos poseen, en su gran mayoría, están muy relacionados con sus estudios y que la cantidad de horas trabajadas excede ampliamente lo recomendado. La alta relación entre estudios y trabajo actúa como un factor motivador dándole la oportunidad al alumno de apropiarse significativamente de los contenidos. También permite que el estudiante desarrolle un aprendizaje integral, mejorando sus oportunidades laborales futuras al contar con experiencia previa en la profesión. Sin embargo, la gran cantidad de horas que trabajan estos estudiantes puede lentificar la finalización de sus estudios y en algunos casos este rezago puede derivar en el abandono.

\footnotetext{
Abstract

The aim of this article is to determine how the simultaneity between higher education and work affects the academic career of the advanced students of the Faculty of Economics of the Universidad Nacional del Litoral (FCEUNL). In order to achieve this goal, information from a 2015 survey of FCE-UNL students and official FCE-UNL data were utilized. This study
} 


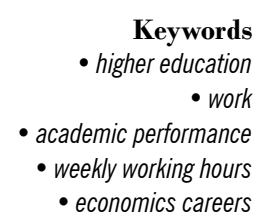

Keywords

- work out the university.

shows that students' jobs are highly related to their studies and that the number of working hours exceeds widely what it is recommended. The high relationship between studies and work acts as a motivating factor, giving the student the opportunity to appropriate the contents significantly. It also allows the student to develop a holistic learning, improving his future job opportunities as it has previous experience in the profession. However, the large number of hours that these students work can slow down the completion of their studies and in some cases this delay can lead to drop

\section{Introducción}

Realizar estudios superiores otorga la posibilidad de profesionalizarse y mejorar las oportunidades de inserción laboral. Para esto, no basta sólo con formarse dentro del aula, sino que también es necesario relacionar esos conocimientos en la práctica. El trabajo brinda esta posibilidad, aunque ocurre que no todos los trabajos le permiten al estudiante hacerlo. Un trabajo relacionado con el área de estudios del alumno podría resultar provechoso para la formación de los sujetos; sin embargo, es necesario reconocer también cómo impactan académicamente en los alumnos aquellos trabajos que no están relacionados con sus estudios. La normativa vigente argentina rescata, en su ley de Sistema de Pasantías Educativas (Ley 26427/08), la importancia de realizar actividades relacionadas con el área de estudios. En dicha norma, en su artículo $2^{\circ}$, se define a las pasantías como el "conjunto de actividades formativas que realicen los estudiantes [...] sustantivamente relacionado con la propuesta curricular de los estudios cursados en unidades educativas, que se reconoce como experiencia de alto valor pedagógico». Asimismo, en el artículo $3^{\circ}$ de la referida norma se plantea como objetivos de dicha experiencia lograr que los pasantes:

a) Profundicen la valoración del trabajo como elemento indispensable y dignificador para la vida, desde una concepción cultural y no meramente utilitaria; b) Realicen prácticas complementarias a su formación académica, que enriquezcan la propuesta curricular de los estudios que cursan;

c) Incorporen saberes, habilidades y actitudes vinculados a situaciones reales del mundo del trabajo;

d) Adquieran conocimientos que contribuyan a mejorar sus posibilidades de inserción en el ámbito laboral;

e) Aumenten el conocimiento y manejo de tecnologías vigentes;

f) Cuenten con herramientas que contribuyan a una correcta elección u orientación profesional futura;

g) Se beneficien con el mejoramiento de la propuesta formativa, a partir del vínculo entre las instituciones educativas y los organismos y empresas referidos en el artículo $1^{0}$ de la presente ley;

h) Progresen en el proceso de orientación respecto de los posibles campos específicos de desempeño laboral.

Por otra parte, el artículo $13^{\circ}$ de la normativa citada limita la cantidad de horas de trabajo de las pasantías a un máximo de 20 horas semanales. Si consideramos que dicho límite existe para permitir que el estudiante pueda trabajar y estudiar simultáneamente evitando que el trabajo sea un obstáculo en el avance de la carrera, aquellos estudiantes que trabajen por encima del límite de 20 horas 
semanales podrán estar en una situación más vulnerable respecto de sus pares que no trabajan 0 que lo hacen durante una cantidad de horas que no excede el límite referido. Así, uno de los datos analizados refiere a la cantidad de horas semanales que los estudiantes de la FCE dedican a sus trabajos. Para esto, se utiliza información estadística proporcionada por el departamento de Alumnado de la FCE-UNL e información primaria a partir de los datos de una encuesta realizada en el 2015 dentro del proyecto de investigación «Estudios Superiores y Trabajo: configuraciones y sentidos desde la mirada de distintos actores de la Facultad de Ciencias Económicas (FCE) de la Universidad Nacional del Litoral (UNL)».

\section{Estudios superiores y trabajo}

\subsection{Relación entre el trabajo y el área de estudios}

La mayor parte de la bibliografía consultada considera que cuando el trabajo está relacionado con el área de estudios el desempeño académico del estudiante tiende a mejorar, siendo también una experiencia provechosa en otros aspectos. Bardagi, Pacheco Lassance, Paradiso y Almeida de Menezes (2006) consideran que aquellos que participan de actividades relacionadas con la profesión cuentan con información más realista y concreta de ésta, lo que permite ratificar la elección de la carrera y afirmar la decisión de entrar en el mercado laboral. Little y Harvey (2006), al entrevistar a estudiantes de siete instituciones de educación superior del Reino Unido, notaron que la mayoría de los estudiantes coincidieron en que el desarrollo personal e interpersonal vivenciado está muy influenciado por la posibilidad de poner en práctica sus saberes. Purcell, Elias, Davies y Wilton (2005), en otro estudio realizado también en Reino Unido, hallaron que los encuestados que realizaron pasantías 0 algún otro tipo de trabajo para ganar experiencia relacionada a la carrera mientras estudiaban fueron, una vez graduados, menos propensos (aproximadamente un tercio menos) a ser empleados en un trabajo en que no se requiere estar graduado, en comparación con aquellos encuestados sin previa experiencia laboral relacionada con el área de estudios. En el mismo sentido, encontraron que los primeros fueron, respecto de los segundos, un $15 \%$ más propensos a ser empleados en trabajos de alta calidad.

Sin embargo, cuando el foco de atención se pone sobre aquellos trabajos que no están relacionados con el área de estudio, las opiniones se dividen. En el estudio de Bardagi et ál. (2006), al exponer aspectos positivos del trabajo sobre el estudiante, sólo se tiene en cuenta aquel que tiene relación con su carrera. Fazio (2004), en su artículo con base en información proveniente del Primer Censo de estudiantes de universidades nacionales argentinas de 1994, concluye que si el trabajo del estudiante no se vincula con su carrera, la relación entre las horas trabajadas y el rendimiento académico será negativa, aún cuando se trabajen pocas horas. Por el contrario, otros autores como Little y Harvey (2006), cuyo estudio parte de entrevistas realizadas a estudiantes de siete instituciones de educación superior del Reino Unido, McInnis y Hartley (2002), en su trabajo referido a estudiantes de universidades australianas y Robotham (2012), que basa su artículo en encuestas realizadas en una universidad del Reino Unido, afirman que aunque no exista esta relación, igualmente el estudiante se beneficia al desarrollar habilidades personales (mayor confianza y mejor administración del tiempo) e interpersonales (comunicación y trabajo en equipo).

Existen estudios que investigan cuántos estudiantes realizan trabajos relacionados con la carrera, y en la mayoría de los casos el porcentaje es bajo. Según un estudio hecho sobre estudiantes de la Universidad Federal de Río Grande do Sul, Brasil (Bardagi et ál., 2006), sólo el 35,4 \% de los alumnos que trabajan lo hacen en un empleo con algún tipo de relación con la profesión (16,14 \% del total de estudiantes). 
Otro estudio hecho en una universidad de Inglaterra (Robotham, 2012) muestra que los trabajos relacionados representan un $1,22 \%$ de los estudiantes trabajadores (0,82\% del total de estudiantes). Callender (2008) concluye que los trabajos de los estudiantes universitarios en Australia no tienen relación con sus estudios y que el $88 \%$ de los estudiantes trabaja en empleos de mano de obra poco o no calificada.

\subsection{Cantidad de horas que trabajan los estudiantes}

Las horas de trabajo de un estudiante impactan en su vida académica. Al respecto existen distintas perspectivas teóricas que analizan qué aspectos de la vida del estudiante afectan y en qué medida. En la mayoría de los casos se manifiesta que las horas de trabajo impactan principalmente en el desempeño académico del estudiante. Con relación a esto, uno de los datos que se busca conocer refiere a cuántas horas trabajan los alumnos. Bexley, Daroesman, Arkoudis y James (2013), en su reporte sobre las universidades australianas, encontraron que el promedio de horas trabajadas semanalmente por estudiantes era de 16 horas. En otro estudio anterior también realizado sobre las universidades australianas, Devlin, James y Grigg (2008) hallaron que dicho promedio era de 14,8 horas. En Reino Unido, un artículo basado en datos provenientes de seis universidades de dicho país (Callender, 2008) reveló un promedio de 15 horas. En el mismo sentido, otro aspecto que suele considerarse es hasta qué punto las horas trabajadas por el estudiante no perjudican su rendimiento académico 0 hasta qué punto lo benefician. Franke (2003) en su investigación basada en estudiantes canadienses de los niveles secundario y terciario, explica que aquellos trabajos de 15 horas semanales o menos no modifican drásticamente el tiempo de los alumnos dedicado a estudiar. Sin embargo, cuando se trata de trabajos de 20 horas semanales 0 más, el impacto negativo es significativo. Siguiendo esta línea, Fazio (2004) en Argentina muestra que en un tramo de baja cantidad de horas (14,5 horas semanales) el rendimiento del alumno puede llegar a ser maximizado si su trabajo tiene cierta relación con sus estudios. Más allá de eso, las horas trabajadas inciden negativamente. No obstante, si el trabajo tiene una fuerte relación con sus estudios, el estudiante se beneficiará académicamente hasta una mayor cantidad de horas trabajadas (24,5 horas semanales).

Por otra parte, existen estudios (aunque en menor cantidad) en que no se verifica que el trabajo impacte realmente en el desempeño académico del estudiante, y/0 se hace un mayor hincapié en el efecto producido en otros aspectos de la vida del estudiante. En un estudio realizado en Estados Unidos (Darolia, 2014) ${ }^{1}$ no se encuentran evidencias significativas para determinar que existe una relación entre las calificaciones y las horas semanales trabajadas de los estudiantes. Nonis y Hudson (2006), a partir de encuestas realizadas a estudiantes de diferentes universidades de negocios de Estados Unidos, determinan que, contrariamente a lo que comúnmente se cree, el tiempo dedicado a trabajar no tiene una incidencia directa con el desempeño académico, sino que los principales factores que afectan dicho desempeño son la capacidad y motivación del alumno. En el mismo sentido, en el trabajo sobre estudiantes de universidades australianas llevado a cabo por McInnis y Hartley (2002) se concluye que muchas o pocas horas de trabajo no tienen un impacto significativo en las calificaciones, sino que son el compromiso académico, la motivación para estudiar y la administración del tiempo, los factores más influyentes.

Asimismo, se destaca el efecto que podrán tener las horas de trabajo en otros aspectos de la vida del alumno si no se logra un balance entre estudios 
y trabajo. Las consecuencias pueden ser serias no sólo en términos de desempeño académico, sino también con respecto al bienestar personal del estudiante. En este estudio se observa que el $39 \%$ de los encuestados coincidía en que el estrés proveniente de las condiciones de trabajo afectaba su capacidad para hacer frente a sus responsabilidades académicas. Del mismo modo, Franke (2003) menciona este tema al aclarar que si bien las horas de trabajo afectan principalmente el tiempo de estudio en Canadá, también afectan tanto a las horas de sueño y ocio, como a factores relacionados con posibles problemas de salud, como el estrés.

Un dato particular es aportado por una investigación realizada en Inglaterra (Purcell, Elias, Davies y Wilton, 2005). Según este estudio, el desempeño académico en aquellos graduados que como estudiantes sólo realizaron trabajos durante el período de vacaciones, no tuvo significativas diferencias respecto del de aquellos que no trabajaron siendo universitarios. Sin embargo, quienes como estudiantes realizaron trabajos durante el período lectivo y en vacaciones, o sólo durante el período lectivo, tuvieron un desempeño académico menor al de sus pares que no trabajaron 0 sólo lo hicieron durante el período de vacaciones.

\section{Estudios y trabajo en la FCE-UNL}

\subsection{Grado de relación entre}

\section{los estudios y el trabajo}

Respecto de la relación entre los estudios y el trabajo, la encuesta realizada muestra que de los estudiantes que actualmente trabajan el $53 \%$ realiza tareas con un alto grado de relación con los estudios, el $26 \%$ lleva a cabo tareas con un mediano grado de relación y el 21 \% lleva adelante tareas con un escaso 0 nulo grado de relación. Sin embargo, si tomamos en cuenta el año de cursado de los encuestados, esta relación se presentará de un modo heterogéneo. En el siguiente gráfico puede observarse que conforme mayor sea el año de cursado mayor es el porcentaje de estudiantes realizando trabajos con un alto grado de relación.

Gráfico 1. Grado de relación en el trabajo según el año de cursado

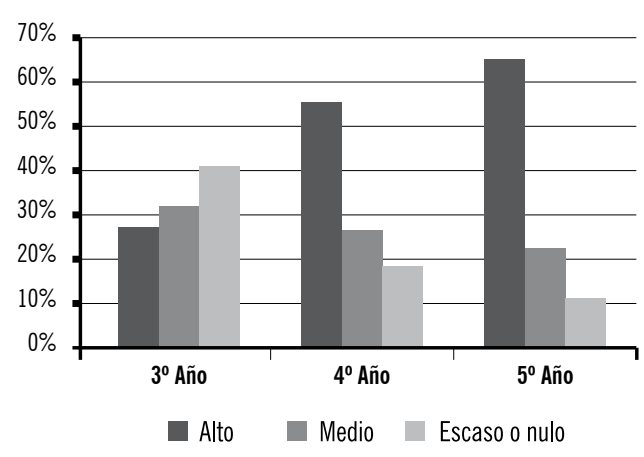

Fuente: Elaboración propia.

En el Gráfico 2 se muestra a los estudiantes que al momento de la encuesta no se encontraban trabajando, pero que han tenido experiencias laborales previas. En este caso notamos que si bien predominan los trabajos con alto grado de relación, la diferencia entre estos y aquéllos de escasa 0 nula es significativamente menor a la existente entre los trabajos de los estudiantes que actualmente trabajan.

Gráfico 2. Grado de relación en trabajos previos de estudiantes que no trabajan actualmente

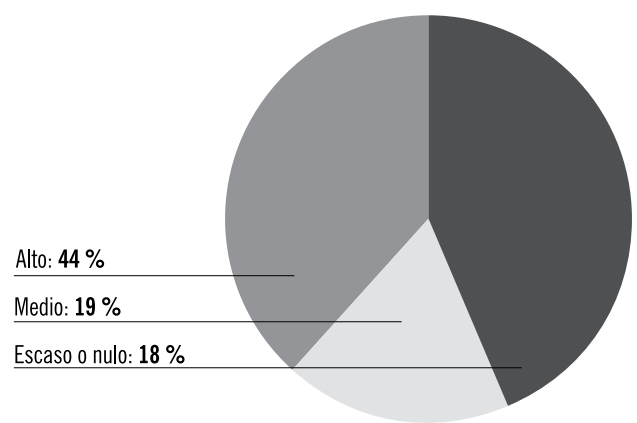

Fuente: Elaboración propia. 


\subsection{Cantidad de horas trabajadas por los estudiantes del Ciclo Especializado}

Según los datos obtenidos del Programa de Información y Análisis Institucional UNL, ${ }^{2}$ del total de estudiantes de la FCE-UNL que trabajan sólo el $33 \%$ Io hace menos de 20 horas a la semana. El $26 \%$ trabaja entre 20 y 35 horas semanales y el $41 \%$ trabaja más de 35 horas semanales. Esto quiere decir que el $67 \%$ de los estudiantes que trabajan lo hacen tanto por encima del límite de 20 horas que el marco legal argentino impone para el régimen de pasantías, como del rango de horas que la literatura recomienda para que el trabajo no perjudique el desempeño académico del estudiante. El promedio de horas trabajadas de los estudiantes de la FCE-UNL se acerca a las 31 horas promedio de trabajo semanales encontradas por Fazio (2004) en su estudio basado en los datos del Censo de Estudiantes de Universidades Nacionales de 1994 de la Secretaría de Políticas Universitarias. En la mayoría de los estudios anglosajones, a la hora de recabar y exponer la información se hace una diferenciación entre los denominados full-time students (estudiantes de tiempo completo) y los part-time students (estudiantes de medio tiempo), distinción que no aparece en los estudios nacionales.

Por otra parte, si analizamos la cantidad de horas que trabajan los estudiantes por carrera, se observará que la tendencia mencionada en el párrafo anterior se mantiene para los estudiantes de las carreras de Contador Público Nacional y Licenciado en Administración, pero en el caso de los estudiantes de la carrera de Licenciado en Economía, dicha tendencia es distinta. Para las carreras de Contador Público Nacional y Licenciado en Administración, más del 40 \% trabaja más de 35 horas semanales, más del $30 \%$ lo hace durante menos de 20 horas semanales y el $25 \%$ entre 20 y 35 horas por semana. Para la carrera de Licenciado en Economía, en cambio, el 38 \% trabaja menos de 20 horas semanales, el $33 \%$ entre 20 y 35 horas por semana y el $29 \%$ durante más de 35 horas semanales. Es importante aclarar que existen diferencias también respecto de la cantidad de estudiantes que trabajan en cada carrera. Mientras que en la Licenciatura en Economía trabaja poco más del $40 \%$, en las dos restantes carreras los estudiantes que trabajan representan aproximadamente el $50 \%$ del total de estudiantes.

\subsection{Interrupción de los estudios}

Del total de estudiantes encuestados, quienes han interrumpido sus estudios representan el $10 \%$, casi en su totalidad (el $95 \%$ ) se trata de aquellos que trabajan 0 han trabajado. Si se toma en cuenta la población estudiantil que trabaja 0 trabajó, el 17 \% de ellos interrumpió sus estudios. Si en cambio se considera a la población estudiantil que no trabaja ni trabajó, el porcentaje que interrumpió sus estudios es el $1 \%$.

Por otra parte, producto de la misma encuesta se observa que cuentan, en promedio, con un mayor número de aplazos quienes trabajan o trabajaron respecto de aquellos que nunca lo hicieron. Para los primeros, la cantidad promedio de aplazos es de 7,52 mientras que para los segundos dicho promedio es de 6,41.

\section{Conclusiones}

Distintos autores a quienes nos hemos referido en el presente estudio sostienen que el número de horas que representan un punto de equilibrio entre el tiempo de estudio y de trabajo se encuentra en el rango de 15-20 horas por semana. Con esto comprobamos que el límite de 20 horas semanales de trabajo que impone la ley del Sistema de Pasantías coincide con la literatura existente sobre el tema. Sin embargo, los datos oficiales de la FCE- 
UNL reflejan que el $67 \%$ de los estudiantes del ciclo superior de esta institución trabaja por encima del rango ideal. Esto implica que pueden verse perjudicadas las horas de cursado, el tiempo dedicado por el estudiante a estudiar y hasta su salud. Algunos de estos perjuicios pueden verse reflejados en datos derivados de la encuesta realizada. Se observa que aquellos estudiantes que trabajan 0 trabajaron se encuentran en una situación más vulnerable respecto de quienes nunca lo hicieron, ya que puede notarse en los primeros una tendencia algo mayor de interrupción de sus estudios y un promedio algo mayor de aplazos.

Por otra parte, respecto de la relación entre el trabajo y los estudios los estudiantes de la FCE-UNL aproximadamente el $80 \%$ muestra un mediano 0 alto grado de relación, lo cual es un aspecto positivo si lo comparamos con la bibliografía internacional a la que se ha hecho referencia con anterioridad, donde la mayoría de los trabajos no tienen relación con el área de estudios del alumno. Empero, esta relación varía según el año de cursado en que se encuentra el alumno, ya que cuanto mayor es el año de cursado, mayor es el grado de relación.

Debe decirse que el alto grado de relación entre los estudios y trabajos de los estudiantes del Ciclo Especializado de la FCE-UNL es un aspecto muy positivo. Por un lado, actúa como un factor motivador al darle al alumno la oportunidad de relacionar ambas prácticas, logrando una mayor comprensión de la profesión. Por otro, permite que el estudiante desarrolle un aprendizaje integral, mejorando sus posibilidades laborales como futuro graduado al contar con experiencia previa en la profesión. № obstante, la gran cantidad de horas que trabajan estos estudiantes es un factor que debe tenerse en cuenta ya que puede lentificar la finalización de sus estudios y, en algunos casos, derivar en el abandono de la carrera.

\section{Referencias bibliográficas}

- Bardagi, M.P., Lassance, M.C.P., Paradiso, A.C. y Menezes, I.A. (2006). Escolha profissional e inserção no mercado de trabalho: percepções de estudantes formandos. Psicologia Escolar e Educacional, 10(1), 69-82. - Bexley, E., Daroesman, S., Arkoudis, S. y James, R. (2013). University student finances in 2012: A study of the financial circumstances of domestic and international students in Australia's universities. Canberra: Universities Australia.

- Callender, C. (2008). The impact of term-time employment on higher education students' academic attainment and achievement. Journal of Education Policy, 23(4), 359-377.
- Darolia, R. (2014). Working (and studying) day and night: Heterogeneous effects of working on the academic performance of full-time and part-time students. Economics of Education Review, 38, 38-50.

- Devlin, M., James R. y Grigg, G. (2008). Studying and working: A national survey of student finances and student engagement. Tertiary Education and Management, 14, 111-122. - Fazio, M.V. (2004, junio). Incidencia de las horas trabajadas en el rendimiento académico de estudiantes universitarios argentinos. Documentos de Trabajo UNLP, 52. Recuperado de http://www.depeco.econo.unlp. edu.ar/doctrab/doc52.pdf
- Franke, S. (2003). Studying and working: the busy lives of students with paid employment. Canadian Social Trends, Statistics Canada, Catalogue 11-008, 22-25.

- Ley 26427 (2008). Sistema de Pasantías Educativas. Boletín oficial de la República Argentina Nro 31557 del 22 de diciembre de 2008. Recuperado de http://servicios.infoleg.gob.ar/infoleglnternet/ anexos/145000-149999/148599/ norma.htm

- Little, B. y Harvey, L. (2006). Learning through work placements and beyond: A report for HECSU and the Higher Education Academy's Work Placements Organisation Fo- 
rum. Centre for Research and Evaluation. Recuperado de http://www. hecsu.ac.uk/assets/assets/documents/Learning_through_work_placements_and_beyond.pdf

- Mclnnis, C. y Hartley, R. (2002). Managing study and work: The impact of full-time study and paid work on the undergraduate experience in Australian universities. Evaluations and Investigations Programme re- port, 02/6. Canberra: Department of Education, Science and Training.

- Nonis, S. y Hudson, G. (2006). Academic performance of college students: influence of time spent studying and working. Journal of Education for Business, 81(3), 151-159.

- Purcell, K., Elias, P., Davies, R. y Wilton, N. (2005). The class of ' 99 . A study of the early labour market experiences of recent graduates. DfES
Research Report No. 691. University of Warwick and University of the West of England. Recuperado de http:// www.hecsu.ac.uk/assets/assets/ documents/Class_99_Full.pdf - Robotham, D. (2012). Student part-time employment: characteristics and consequences. Education \& Training, 54(1), 65-75.

\section{Registro bibliográfico}

Pacífico, A., y Gandolfo, P. H. (2016).

Estudios superiores y trabajo: implicancias

de su simultaneidad. Revista Ciencias

Económicas, 13(01), 153-160. 